\title{
Inteligencia emocional y tutoría docente en prácticantes pre profesionales de una universidad estatal limeña
}

\author{
Emotional intelligence and teaching tutoring in pre-professional interns- \\ hips at a limeña state university
}

\author{
Nilda Florian Angulo ${ }^{1}$ \\ MENTEM
}

Recibido: $14-11-20$

Aceptado: $16-11-20$

Publicado: $21-12-20$

\section{Resumen}

La investigación que presentamos, nace desde el interés de mejorar las capacidades relacionadas a las prácticas pre profesionales, las mismas que nos preparan para desenvolvernos como profesionales docentes al termino de los estudios universitarios, hemos observado desde nuestra experiencia universitaria alumnos que muestran inseguridad y vacíos durante el actuar en los mencionados cursos relacionados a las prácticas mencionadas, el estudio se realiza en estudiantes del ultimo año de formación en educación en una universidad pública de Lima. La muestra fue $40 \%$ del porcentaje total de estudiantes (122 futuros profesionales del X semestre del curso de práctica pre profesional). La presente investigación tiene por objetivo llegar a conocer e identificar de forma real y objetiva la relación entre tutoría docente con el intelecto emocional en los practicantes pre profesionales que están por egresar. Por las características y función del presente estudio es de corte cuantitativo, descriptivo correlacional de tipo transversal. Usamos el Test REUVEN BAR-ON, para la recolección de datos para la variable X, relacionada con la I.E, así como se utilizó un cuestionario LIKERT para la variable Y relacionada a la tutoría docente.

Según los datos estadísticos del estudio planteado, confirmamos la hipótesis general, determinando una relación significativa entre el acompañamiento docente e inteligencia emocional en los estudiantes de practica pre profesional en cuestión. Se concluye que existen algunas divergencias y carencias en cuanto al aspecto emocional y las habilidades profesionales - pedagógicas, en los practicantes de último ciclo; dado que no expresan respuestas contundentes.

Palabras claves: Tutoría docente; inteligencia emocional; adaptabilidad; habilidades profesionales. manejo de estrés.

1 Docente y directora en MENTEM.

E-mail: floriannilda77@gmail.com ORCID: https://orcid.org/0000-0001-5000-3494

(C) Los autores. Este artículo es publicado por la Revista de Investigación en Psicología de la Facultad de Psicología, Universidad Nacional Mayor de San Marcos. Este es un artículo de acceso abierto, distribuido bajo los términos de la licencia Creative Commons Atribucion - No Comercia_Compartir Igual 4.0 Internacional. (http://creativecommons.org/licenses/by-nc-sa/4.0/) que permite el uso no comercial, distribución y reproducción en cualquier medio, siempre que la obra original sea debidamente citada. 


\begin{abstract}
The research we present is born from the interest of improving the capacities related to preprofessional practices, the same ones that prepare us to function as teaching professionals at the end of university studies, we have observed from our university experience students who showed insecurity and emptiness during Acting in the aforementioned courses related to the aforementioned practices, the study is carried out in students of the last year of education in a public university in Lima. The sample was $40 \%$ of the total percentage of students (122 future professionals from the $\mathrm{X}$ semester of the pre-professional practice course). The present research aims to get to know and identify in a real and objective way the relationship between teacher tutoring with the emotional intellect in pre-professional practitioners who are about to graduate. Due to the characteristics and function of this study, it is quantitative, descriptive, correlational, cross-sectional. We used the REUVEN BAR-ON Test, for data collection for variable $\mathrm{X}$, related to the E.I, as well as a LIKERT questionnaire for variable Y related to teacher tutoring.
\end{abstract}

According to the statistical data of the proposed study, we confirm the general hypothesis, determining a significant relationship between the teaching accompaniment and emotional intelligence in the pre-professional practice students in question. It is concluded that there are some divergences and shortcomings in terms of the emotional aspect and the professional pedagogical skills, in the last cycle practitioners; since they do not express forceful answers.

Keywords: Teacher tutoring; emotional intelligence; adaptability; professional skills. stress management.

Es importante y necesario saber, qué tanto se está avanzando en el desarrollo integral del estudiante a nivel superior, si bien es cierto, los alumnos de practica pre profesional, los que ya están por egresar cuentan con un tutor docente que velará por su desempeño académico, emocional y profesional también existe la interrogante de cómo se viene llevando a cabo el acompañamiento docente en este nivel, con los futuros profesores y qué características debe poseer una persona emocionalmente inteligente. En los centros de formación existen ciertas competencias que mejoran el actuar del grupo humano que no han sido valoradas tradicionalmente, "existen un conjunto de competencias y destrezas personales, emocionales y sociales que favorecen las estrategias de afrontamiento y condicionan nuestra eficacia para comprender y relacionarlos con los demás, así como para resolver problemas del día a día" (Molero, Pantoja y Galiano, 2017, p 45) "La inteligencia emocional se puede aprender y así controlar emociones tan significativas en el mundo educativo como la ansiedad, las presiones, los miedos (estrés) y la agresividad" (Ros, Moya y Garcés, 2013, p.106) Sabemos que este último ciclo de formación simboliza un punto tan relevante en los estudiantes en formación y muchas veces estos jóvenes pasan por muchas incertidumbres, porque no saben cómo efectuar de manera exitosa sus prácticas y convertirse en un buen profesional (Galaz, 2011, p. 48). Es preciso mencionar al director de Salud Mental del Ministerio de Salud, Cutipé (2019), quien indicó que, según resultados obtenidos en otros centros universitarios, más del $30 \%$ de alumnos debe sufrir de 
algún desorden o trastorno mental. Indicó también que entre los trastornos más comunes se encuentran los depresivos, incluso con ideas de suicidio, y problemas por consumo de alcohol. Es claro que nos encontramos ante nuevas necesidades formativas, y nos urge desarrollar nuevas competencias en el campo educacional. Asimismo, cabe resaltar a Guerra y Villarroel (2010), quienes aseveran sobre las condiciones intelectuales, que no son precisamente garantía de éxito en nuestras labores profesionales; puesto que en la vida profesional también se nos juzga cómo nos relacionamos con los demás y que no solo cuenta nuestro bagaje académico. En efecto Bar-On (2006) sostiene que el intelecto emotivo es una relación entre habilidades emocionales y competencias y personales que determinan las habilidades generales, las cuales suman a nuestra salud emocional. Estas herramientas y comportamientos sociales ayudaran también cumplir los retos y demandas, de nuestro quehacer diario. Como vemos, es conveniente y necesario que en la etapa formativa el futuro educador reciba los conocimientos e instrumentos pertinentes y la orientación adecuada para consolidar su personalidad y así pueda actuar con eficiencia, eficacia y satisfacción en los practicantes profesionales y posteriormente actúe en coherencia en el ejercicio de su profesión. "la capacidad de auto motivarnos, de sostener el empeño a pesar de frustraciones posibles, de controlar los impulsos, saber manejar las gratificaciones, regular nuestros propios estados de ánimo, no permitir que la angustia interfiera con nuestras facultades racionales, así como poseer la capacidad de empatía y confianza en los demás "Goleman (1995, p. 35). Coincidimos con Lázaro (2007) sobre la importancia de contar con una Tutoría diferenciada, pues este planteamiento nos lleva a una cuestión más específica: es necesario un respectivo tutor que obedezca tanto al nivel de lo que se requiere aprender, así como la edad del practicante. El tutor universitario no puede ser el mismo que el tutor de niveles anteriores como es el de Primaria y Secundaria. (p. 5). Asimismo, García y Nora (2010) “competencias básicas” indican que las funciones del tutor docente deben poseer características mediacionales, intentando siempre de buscar alternativas que faciliten la permanencia del dicente practicante en la carrera, así como adaptarse y saber sortear ciertas dificultades (P. 5). Álvarez (2002, p. 34) determina "dichos tutores deberán compaginar y globalizar los conocimientos pertinentes y significativos en una forma estructurada y certera para que se cumpla el proceso formativo en el dicente practicante". Según diversos autores el perfil debe ajustarse a dos criterios: el que atañe al factor de su evolución personal y el que se refiere al desempeño profesional en la enseñanza como tutordocente. El autoconcepto, la autoestima y las relaciones humanas equilibradas son prescindibles para el autodesarrollo de los estudiantes. Además, no se puede tener certeza del apoyo interno del alumno cuando nosotros estamos desequilibrados o desechos (p.138) "la influencia de las emociones en complejos procesos cognitivos como: el razonamiento, la creatividad, el aprendizaje y la toma de decisiones, son indispensables en el estudio de cualquier fenómeno" (Fragoso, 2019, p. 3). La mayoría de autores determinan que la persona con intelecto emotivo es consciente de sí 
mismo y conduce su vida sin que las emociones la alteren. Para el mencionado autor la motivación, comprensión hacia los demás, así como la disciplina y constancia son determinantes "los futuros docentes con mayor responsabilidad, optimismo y extraversión indicadores de inteligencia emocional tendrán mayores niveles de satisfacción vital" (Cazalla y Molero, 2015, p. 241). Por otro lado, muchos autores determinan la importancia del desarrollo de la inteligencia emocional en el ámbito educativo "La Inteligencia emocional es un factor que influye positivamente en el bienestar mental y social de las personas, asimismo dentro del ámbito educativo posee un papel fundamental en el proceso de enseñanza-aprendizaje" (Puertas, Ubago, Molero, Padial, Martínez y Gonzáles, 2018, p. 1). Los mismos autores determinan que el desarrollo de las competencias profesionales en educación por sus características debe desarrollarse la inteligencia emocional "Las competencias de los profesionales de la educación, el contacto social en el entorno educativo y la resolución de conflictos, son factores que manifiestan la necesidad de desarrollar la Inteligencia Emocional" (Puertas et al., 2018). La finalidad de este estudio reside en realizar una revisión sistemática que aborde la Inteligencia Emocional como factor en el desempeño de los docentes en las diferentes etapas educativas, para dar solución a esta preocupación del ámbito educativo, es importante generar las condiciones para el desarrollo de la autonomía y otros aspectos importantes como o menciona (Martínez, 2016, p .9) ) "El estudiante con autonomía, con perspectiva de poderlo hacer a lo largo de su vida, en contextos de cooperación y construcción colectiva de conocimiento; y se expresen en toda su riqueza, aprendan a valorar, a tener criterio propio, a argumentar con rigor y fundamento, a saber convivir en un mundo plural y a estimar los valores propios de una sociedad basada en el respeto y la dignidad de todas las personas" Es preciso resaltar que uno de los vacíos que se pudo observar en el proceso de nuestra investigación es que no existen muchos conceptos sólidos ni materiales contundentes sobre lo que realmente significa ser un tutor docente en el acompañamiento tutorial de los estudiantes practicantes en el nivel superior.

\section{Antecedentes de investigación nacional e internacional}

Espinosa (2013), en la investigación "La inteligencia emocional del docente de Química del nivel superior como factor para el desempeño académico de sus alumnos" tesis para obtener el grado de Doctor en educación incluye una muestra de 92 docentes, así como 120 estudiantes, concluye una relación alta entre la inteligencia emocional docente y el aprendizaje de los estudiantes.

Pérez (2012), trabajo para obtener el grado de doctor "inteligencia emocional y motivación del estudiante universitario". Formula que el clima motivacional mediaría con la "percepción de las necesidades psicológicas básicas (autonomía, relaciones y competencia)". Los datos estadísticos infieren una relación significativa entre clima motivacional y las necesidades psicológicas estudiadas afirmando la hipótesis inicial. "Cuando el profesorado es capaz de conocer el 
desarrollo emocional de su alumnado tiene una información muy valiosa para averiguar el estado de bienestar de los mismos"

Damián y Villarroel (2010), presenta un estudio denominado "inteligencia emocional y desempeño docente en el proceso de enseñanza- aprendizaje", tesis de maestría de la Universidad Nacional de Educación Enrique Guzmán y Valle. Contó con una población y muestra de 38 profesores, realizó una estadística inferencial descriptiva. Determina que los "individuos hábiles en la percepción y comprensión de emociones ajenas son inteligentes emocionales" desarrollan más habilidades de percepción y comprensión, así como el manejo de emociones en los otros. Siendo importante para la comprensión del actuar docente que muchas veces no cuenta la experiencia o formación sino la asertiva relación con nuestro entorno.

Coronel y Ñaupari (2011) Estudian la relación entre la inteligencia emocional y su relación con la variable desempeño docente es estudio se realiza en la Universidad Continental, Concluyendo que hay una relación, significativa y directa en ambas variables desempeño docente en el aula e inteligencia emocional.

Bazán (2012), presenta una tesis que lleva el título de "La inteligencia emocional y su relación con el nivel de logro en el área de personal social de las niñas $y$ niños de 5 años" determina el estudio usando 120 niños como población y 59 niños de muestra concluyendo que el mal manejo de emociones genera conflictos relacionados a la disminución de la productividad.

\section{METODOLOGIA}

Tipo y diseño de investigación: El presente trabajo es de diseño descriptivo- correlacional. Asimismo, es de enfoque cuantitativo y de tipo transversal, puesto que el estudio se caracteriza porque la información con respecto a cada unidad de análisis se recogerá en un solo momento.

\section{Muestra}

Básicamente la población se obtuvo de estudiantes de educación del último semestre de estudios quienes ejercían su respectiva practica pre profesional. Lo cual atribuía a una población de 295 estudiantes, 176 de educación y 119 de educación física respectivamente. Para Hernández (2014, p. 174) "una población es el conglomerado de todos los casos que convergen con sus respectivas especificaciones". Se utilizó un muestreo probabilístico; dado que según el mismo autor indica como muestra: "en esencia, a una subdivisión de la Población. Entendiéndose entonces que es un subconjunto de elementos que pertenecen a esa subdivisión con características definidas al que denominamos población" Dado que se trabajó con el $40 \%$ de la población la muestra fue de 122 estudiante, específicamente 72 estudiantes de educación tanto del nivel inicial, primario y secundario, y 50 alumnos de educación física. 


\section{Instrumentos}

Se empleo para nuestro trabajo el test de Bar- on, para medir el intelecto o control emocional de los respectivos tutores docentes, con su determinada escala de valores de: nunca, pocas veces, a veces, muchas veces y siempre. Asimismo, se elaboró un cuestionario de Likert de 30 preguntas cerradas para conocer la apreciación que los practicantes tienen de sus tutores. Cuestionario dispuesto en escala de valores de nunca a veces y siempre.

Tanto el Test de Bar-on como el cuestionario Likert obedecieron a ciertos componentes, nos referimos a las dimensiones intra personales, interpersonales, manejo de estrés, adaptabilidad o ajuste y estado de ánimo. Todo esto correspondiente a la variable inteligencia emocional. Mientras que los aspectos de habilidades pedagógicas, profesionales y competencias actitudinales; referentes a la variable de Tutoría docente.

\section{Validación de los instrumentos}

Test REUVEN BAR-ON usado en la variable inteligencia emocional, desarrollamos para la variable tutoría docente un cuestionario, el mismo que sometimos a validación.

Se observa en el cuadro anterior que la validación de los expertos arroja un puntaje total de 95\%. Determinando que la aplicación del mismo es recomendable y adecuado (ver Tabla 1).

Tabla 1

Juicio de expertos variable inteligencia emocional.

\begin{tabular}{lll}
\hline $\mathbf{N}^{\circ}$ & Peritos & Estimación \\
\hline 1 & Freddy Huamaní A & $90 \%$ \\
2 & Victoria LLaja R. & $97.5 \%$ \\
3 & Máximo Perez Z. & $97.5 \%$ \\
\hline & Total & $95 \%$ \\
\hline
\end{tabular}

En cuanto a la fiabilidad se aplicó el alfa de Cronbach cuyos resultados arrojaron un ,944 determinando una alta confiabilidad (ver Tabla 2).

Tabla 2

Confiabilidad y fiabilidad

\begin{tabular}{cc}
\hline \multicolumn{2}{c}{ Estadísticas de fiabilidad } \\
\hline Alfa de Cronbach & N de elementos \\
\hline, 944 & 3 \\
\hline
\end{tabular}




\section{Procedimiento}

Se procedió solicitando los respectivos permisos a las autoridades pertinentes, así como a docentes y estudiantes en cuestión. Posteriormente se informó a los involucrados el objetivo de la presente investigación, asimismo las características y propósitos de los instrumentos que se usarían. Los tiempos requeridos para desarrollar o hacer uso de los instrumentos fueron acordados con los practicantes en cuestión. Los datos fueron recibidos en momentos acordados de clase. También contamos con un instrumento guía de observación. La misma que nos permitió, acceder a información que nos ayudaba a conocer cómo abordar y encuadrar nuestro trabajo; de esta manera acudíamos a dicha guía antes de cada paso. Podemos observar en la tabla de correlación un aproximado de 93.7\%, en Rho de Spearman mostrando unas estimaciones cercanas a 1: resaltando un enlace positivo y significativo. Aceptándose así la hipótesis alterna.

\section{RESULTADOS}

Para el tratamiento estadístico, utilizamos el coeficiente de correlación Rho Spearman. En cuanto a la hipótesis general HGA, los resultados arrojaron que la inteligencia emocional se asocia significativamente con el acompañamiento docente en los alumnos practicantes. Vemos un 91.7 concordando en valores próximos a 1: indicando así una correspondencia positiva y significativa. Admitiéndose la hipótesis alterna, que determina la correlación significativa entre el "manejo emocional y tutoría docente" (ver Tabla 3).

Tabla 3

Correlación de la hipótesis general

\begin{tabular}{|c|c|c|c|c|}
\hline \multicolumn{3}{|c|}{ CORRELACION } & $\begin{array}{l}\text { INTELIGENCIA } \\
\text { EMOCIONAL }\end{array}$ & $\begin{array}{l}\text { TUTORIA } \\
\text { DOCENTE }\end{array}$ \\
\hline \multirow{6}{*}{$\begin{array}{l}\text { Rho de } \\
\text { Spearman }\end{array}$} & \multirow{3}{*}{ INTELIGENCIA EMOCIONAL } & Coeficiente de correlación & 1,000 &, $917^{* *}$ \\
\hline & & Sig. (bilateral) & . &, 000 \\
\hline & & $\mathrm{N}$ & 122 & 122 \\
\hline & \multirow{3}{*}{ TUTORIA DOCENTE } & Coeficiente de correlación &, $917^{* *}$ & 1,000 \\
\hline & & Sig. (bilateral) & ,000 & . \\
\hline & & $\mathrm{N}$ & 122 & 122 \\
\hline
\end{tabular}

**. La correlación es significativa al nivel 0,01 (bilateral).

La primera hipótesis específica H1: guarda relación significativa y positiva entre inteligencia intrapersonal y la tutoría docente con respecto a los practicantes pre profesionales. Correlación determina un $90.6 \%$, de C. Rho de Spearman. (ver Tabla 4) 
Tabla 4

Correlación de la primera hipótesis especifica

\begin{tabular}{lllcc}
\hline \multicolumn{2}{c}{ CORRELACION } & INTRAPERSONAL & $\begin{array}{c}\text { TUTORIA } \\
\text { DOCENTE }\end{array}$ \\
\hline \multirow{3}{*}{$\begin{array}{l}\text { Rho de } \\
\text { Spearman }\end{array}$} & INTRAPERSONAL & Coeficiente de correlación & 1,000 &, $906^{* *}$ \\
\cline { 3 - 5 } & & Sig. (bilateral) & $\cdot$ &, 000 \\
\cline { 2 - 5 } & $\mathrm{N}$ & 122 & 122 \\
\cline { 2 - 5 } & TUTORIA DOCENTE & Coeficiente de correlación &, $906^{* *}$ & 1,000 \\
\cline { 3 - 5 } & & Sig. (bilateral) &, 000 & \multicolumn{1}{c}{122} \\
\cline { 2 - 5 } & $\mathrm{N}$ & 122 & \\
\hline
\end{tabular}

**. La correlación es significativa al nivel 0,01 (bilateral).

Contrastación de la segunda hipótesis específica H2: el manejo interpersonal y el acompañamiento docente se relacionan significativamente, en el despliegue de las prácticas de los estudiantes de último ciclo. (ver Tabla 5)

Tabla 5

Correlación de la segunda hipótesis específica

\begin{tabular}{|c|c|c|c|c|}
\hline \multicolumn{3}{|c|}{ CORRELACION } & INTERPERSONAL & $\begin{array}{l}\text { TUTORIA } \\
\text { DOCENTE }\end{array}$ \\
\hline \multirow{6}{*}{$\begin{array}{l}\text { Rho de } \\
\text { Spearman }\end{array}$} & \multirow{3}{*}{ INTERPERSONAL } & $\mathrm{CC}$ & 1,000 & $0,937^{* *}$ \\
\hline & & Sig. (b) & . & 0,000 \\
\hline & & $\mathrm{N}$ & 122 & 122 \\
\hline & \multirow{3}{*}{ TUTORIA DOCENTE } & C.C &, $0937^{* *}$ & 1,000 \\
\hline & & Sig. (b) &, 000 & . \\
\hline & & $\mathrm{N}$ & 122 & 122 \\
\hline
\end{tabular}

**. La C es significativa al nivel de 0,01 (bilateral).

La hipótesis específica $\mathrm{H} 3$, señala una relación positiva directa y significativa con el manejo de estrés y el acompañamiento pedagógico de estudiantes en cuestión. Existe una correlación de 91.6\%, concordando valores próximos a 1: vemos entonces una relación positiva, así como fuerte. (ver Tabla 6)

Tabla 6

Correlación de la tercera hipótesis especifica

\begin{tabular}{|c|c|c|c|c|}
\hline \multicolumn{3}{|c|}{ CORRELACION } & M. ESTRÉS & TUTORIA D. \\
\hline \multirow{6}{*}{$\begin{array}{l}\text { Rho de } \\
\text { Spearman }\end{array}$} & \multirow{3}{*}{ M. ESTRÉS } & Coeficiente de correlación & 1,000 &, $916^{* *}$ \\
\hline & & Sig. (b) & . & 0,000 \\
\hline & & $\mathrm{N}$ & 122 & 122 \\
\hline & \multirow{3}{*}{ TUTORIA DOCENTE } & Coeficiente de correlación &, $916^{* * *}$ & 1,000 \\
\hline & & Sig. (b) &, 000 & . \\
\hline & & $\mathrm{N}$ & 122 & 122 \\
\hline
\end{tabular}

**. La C. es significativa al nivel de 0,01 (bilateral). 
En cuanto a la cuarta hipótesis específica H4: el Estado de ánimo se relaciona significativamente con la tutoría docente se relacionan significativamente en los estudiantes practicantes. Los datos de la tabla determinan un $92.2 \%$, aquí también resulta una correlación positiva y significativa, basados en coeficiente de Rho de Spearman (ver Tabla 7)

Tabla 7

Correlación de la cuarta hipótesis específica

\begin{tabular}{|c|c|c|c|c|}
\hline \multicolumn{3}{|c|}{ CORRELACIÓN } & $\begin{array}{c}\text { ESTADO DE } \\
\text { ANIMO }\end{array}$ & $\begin{array}{l}\text { TUTORIA } \\
\text { DOCENTE }\end{array}$ \\
\hline \multirow{6}{*}{$\begin{array}{l}\text { Rho de } \\
\text { Spearman }\end{array}$} & \multirow{3}{*}{ ESTADO DE ANIMO } & Coeficiente de correlación & 1,000 & $922^{* *}$ \\
\hline & & Sig. (bilateral) & . &, 000 \\
\hline & & $\mathrm{N}$ & 122 & 122 \\
\hline & \multirow{3}{*}{ TUTORIA DOCENTE } & Coeficiente de correlación & $922^{* *}$ & 1,000 \\
\hline & & Sig. (bilateral) &, 000 & . \\
\hline & & $\mathrm{N}$ & 122 & 122 \\
\hline
\end{tabular}

**. La correlación es significativa al nivel 0,01 (bilateral).

En cuanto a la H5: aceptamos la hipótesis alterna, existe una aproximación de $92.6 \%$, señalando así una correlación positiva y significativa entre adaptabilidad y tutoría docente (ver Tabla 8)

Tabla 8

Correlación de la quinta hipótesis especifica

\begin{tabular}{|c|c|c|c|c|}
\hline \multicolumn{3}{|c|}{ CORRELACION } & $\begin{array}{l}\text { ADAPTABILI- } \\
\text { DAD O AJUSTE }\end{array}$ & $\begin{array}{l}\text { TUTORIA } \\
\text { DOCENTE }\end{array}$ \\
\hline \multirow{6}{*}{$\begin{array}{l}\text { Rho de } \\
\text { Spearman }\end{array}$} & \multirow{3}{*}{$\begin{array}{l}\text { ADAPTABILIDAD O } \\
\text { AJUSTE }\end{array}$} & Coeficiente de correlación & 1,000 &, $926^{* *}$ \\
\hline & & Sig. (bilateral) & . &, 000 \\
\hline & & $\mathrm{N}$ & 122 & 122 \\
\hline & \multirow{3}{*}{ TUTORIA DOCENTE } & Coeficiente de correlación & $0,926^{* *}$ & 1,000 \\
\hline & & Sig. (b) & 0,000 & . \\
\hline & & $\mathrm{N}$ & 122 & 122 \\
\hline
\end{tabular}

**. La C es significativa al nivel de 0,01 (b).

\section{DISCUSIÓN}

En el presente trabajo vemos que se corresponden estadísticamente las dimensiones de la tutoría docente e inteligencia emocional, puesto que comprobamos que los investigados no han introyectado elementos mentales, conductuales y emocionales necesarios para su día a día; aquí observamos que el impacto de la tutoría docente no es determinante dado que dentro de la escala de valores la apreciación es de regular. Similares trabajos al nuestro se resaltan en Pérez. (2012), concluye que "el clima motivacional orientado a la tarea predecía positiva y significativamente 
las tres necesidades psicológicas básicas (competencia, autonomía y relaciones), desde luego, podemos ver que el aspecto relacional es de suma importancia, más aún cuando en el campo académico se requiere de un docente tutor que satisfaga las demandas educativas, psicológicas y profesionales".

Nuestro resultado estadístico en cuanto a la hipótesis específica número uno existe una relación significativa entre inteligencia intrapersonal y tutoría docente. Cabe resaltar a Zárate (2012), concluye que la incapacidad para dominar las emociones, genera en las organizaciones conflictos que producen una pérdida de la moral laboral y una disminución de la productividad en el personal. En esta línea podemos ver semejanzas a nuestra hipótesis validada, dado que se observó en cuanto a la variable tutoría docente, siendo más del $50 \%$ de encuestados que poseen competencias actitudinales frente a un $36 \%$ de solo a veces en la escala de valores, en tanto que en la inteligencia intrapersonal se nota una clara divergencia.

En la segunda hipótesis específica representada por la inteligencia interpersonal y su relación con la tutoría docente, podemos indicar que, si existe una correlación fuerte y positiva, pues con respecto a la dimensión Interpersonal, se observa que el $25 \%$ que a veces y $23 \%$ siempre. En cuanto a tutoría docente se aprecia que son pocos los que han incorporado contundentemente las habilidades profesionales. Un estudio similar encontramos en Guerra y Villarroel (2010). Quienes concluyen en su estudio que una persona con alta inteligencia emocional, es una persona más hábil en la percepción y comprensión de emociones ajenas y posee mejores habilidades de percepción, comprensión y manejo de las emociones de los demás.

Para nuestra tercera hipótesis específica, la cual incluye manejo de tensión y su relación con la tutoría docente, cabe mencionar un trabajo similar de Coronel y Ñaupari (2011), donde se concluye que existe una correlación alta, directa y significativa entre las variables inteligencia emocional y desempeño docente en el aula, en la Universidad Continental de Huancayo. En efecto se aprecia en nuestro trabajo de investigación la correlación importante entre las dos variables, dado que los sujetos estudiados han incorporado habilidades docentes y profesionales solo en un porcentaje regular frente a un $24 \%$ que carece de estas habilidades asimismo se recogió divergencias en cuanto al aspecto de inteligencia emocional.

En nuestra cuarta hipótesis específica se indica que entre el estado de ánimo y la tutoría docente existe una correlación significativa. No es tan alentador saber que solo una cuarta parte de encuestados, manejan con propiedad la dimensión de estado de ánimo, Asimismo se halló una correlación fuerte y positiva relacionada a la aptitud de adaptabilidad con tutoría docente en los estudiantes de práctica pre profesional. Observamos que solo a veces la aptitud de adaptabilidad está presente en ellos, frente a un $21 \%$ que expresa ya tenerlo incorporado. 


\section{CONCLUSIONES}

- Según resultados en base al test usado para medir la I.E un $27 \%$ se maneja esta variable de manera equilibrada., siendo la variable tutoría docente regular con un $39 \%$, así como un $43 \%$ indico tener habilidades respectivas relacionadas a tutoría docente.

- Se determinó la existencia de una relación entre la inteligencia intrapersonal y tutoría docente. La población estudiada; no poseen respuestas determinantes; observamos según los datos estadísticos divergencias en sus opiniones, solo un $25 \%$ afirma poseerlo. Por otro lado, se carece de respuestas adaptativas, deducimos, los estudiantes protagonistas en nuestro estudio, no manejan bien los conflictos en la práctica pre profesional; siendo un componente interpersonal es preocupante, ya que determina la interrelación asertiva las personas de su entorno, relacionados con la empatía. Con respecto a las habilidades pedagógicas y profesionales de la tutoría docente se encuentra discrepancias.

- Existe una correlación directa y significativa entre la tutoría y el manejo de la tensión, se observa que el $28 \%$ de sujetos estudiados a veces cuenta con el manejo o tolerancia al estrés, y el $20 \%$ indica que realmente lo posee.

- Menos de la mitad de la población estudiada cuenta con la habilidad para adaptarse a los cambios y resolver problemas de naturaleza personal y social. En cuanto a adaptabilidad el $25 \%$ de los sujetos investigados pocas veces regulan, comportamientos, pensamientos, así como sus sentimientos en situaciones de variabilidad, solo el $21 \%$ afirma tener la capacidad.

\section{RECOMENDACIONES}

- El estado debe generar planes formativos a través del Ministerio de Educación que permitan fortalecer la inteligencia emocional en las personas que participan en el rubro docente, por otro lado, brindar en los planes de estudio de centros de formación inicial docente Institutos pedagógicos y universidades conocimientos específicos relacionados a la inteligencia emocional ventajas y desventajas emotivas.

- El desarrollo de la inteligencia emocional en los estudiantes que formaran futuros ciudadanos debe ser imperativo, ya que dependerá de nuestras prácticas pedagógicas el éxito del aprendizaje de sus futuros estudiantes creando dentro de los planes de estudios ejes transversales para el desarrollo $\mathrm{y}$ equilibrio de las emociones.

- Si bien es cierto la inteligencia emocional se desarrolla a lo largo de nuestra vida y la interacción y las relaciones constituyen factores importantes en el desarrollo de diversos ámbitos de la dimensión humana, creemos que 
el equipo docente y sus directivos deben de generar cambios en los planes de estudio incluyendo competencias que relacionen aspectos humanísticos, relacionadas al trabajo colaborativo y en equipo, convivencia asertiva donde la propuesta de situaciones reales contextualizadas mejoren las capacidades docentes de los estudiantes.

- El conocimiento de estrategias para el pertinente manejo de las emociones debe ser considerado en el quehacer profesional de los que forman futuros maestros dotando al estudiante de herramientas que le permitan superar problemáticas que se dan en la práctica preprofesional siendo una necesidad primordial como lo expresamos anteriormente un reajuste de la malla curricular determinando competencias pertinentes para el desarrollo de relacionadas a habilidades blandas.

- La acción tutorial debe atenuar, fortalecer y mejorar el manejo de las emociones de los estudiantes, logrando así desarrollar confianza, libertad en las construcciones de sus habilidades pedagógicas para efectuar un adecuado desarrollo profesional.

\section{Agradecimientos / Acknowledgments:}

Se agradece la colaboración de la universidad autoridades, estudiantes y profesores que participaron en este estudio.

\section{Fuentes de financiamiento / Funding:}

Recursos propios

\section{Aspectos éticos / legales; Ethics / legals:}

Declaro haber actuado éticamente según los códigos de investigación.

\section{Conflicto de intereses / Competing interests:}

No se ha presentado conflictos de intereses en el estudio y la elaboración de la presente comunicación científica.

\section{REFERENCIAS}

Álvarez P. (2002). La función tutorial en la Universidad. Una apuesta en la mejora de la calidad de enseñanza. Madrid: EOS Universitaria.

Bar-On, R. (2006). The Bar-On model of emocional-social intelligence (ESI). Psicothema, 18, 124-131. Recuperado de http://www.psicothema.com/pdf/3271.pdf

Bazán J. (2012). La inteligencia emocional y su relación con el nivel de logro en el área de personal social de las niñas y niños de 5 años del jardín de niños $N^{\circ} 215$ Trujillo 2012 (Tesis de maestría). Universidad Cesar Vallejo, Trujillo. 
Cazalla-Luna, N., \& Molero, D. (2015). Inteligencia emocional percibida, disposición al optimismo-pesimismo, satisfacción vital y personalidad de docentes en su formación inicial Revista De Investigación Educativa, 34(1), 241-258. https://doi.org/10.6018/ rie.34.1.220701

Cazalla-Luna, N.; Molero, D. (2018). Emociones, afectos, optimismo y satisfacción vital en la formación inicial del profesorado. Profesorado. Revista de Curriculum y Formación del Profesorado, 22 (1), pp. 215-233. http://hdl.handle.net/10481/52484

Coronel, E y Ñaupari, F. (2011). Relación entre inteligencia emocional y desempeño docente en aula en la Universidad Continental de Huancayo. Apunt. Cienc. Soc, 01(01), 27-31 DOI: https://doi.org/10.18259/acs.2011006

Cutipé, Y. (2019). UNMSM: Un tercio de estudiantes sufriría algún trastorno mental. Obtenido de La República: https://larepublica.pe/sociedad/1320645-unmsm-tercioestudiantes-padece-algun-trastorno-mental-afirma-ministerio-salud-minsa-universidad-nacional-mayor-san-marcos-salud-mentalorestes-cachay-yuri-cutipe-examenadmision

Espinosa M. (2013). La inteligencia emocional del docente de química del nivel medio superior como factor para el desempeño académico de sus alumnos, (tesis para optar el grado de doctor). Universidad Iberoamericana, México, D.F.

Encinas V, Carmen R. (2013). Tutoría académica y relación con la reflexión de habilidades metacognitivas en estudiantes de estudios generales de la Universidad de San Martín de Porres (Tesis para optar el grado académico de doctor en educación). Universidad San Martin de Porres, Lima.

Fragoso-Luzuriaga, Rocío. (2019). Importancia del desarrollo de la inteligencia emocional en la formación de personas investigadoras. Actualidades Investigativas en Educación, 19(1), 655-679. https://dx.doi.org/10.15517/aie.v19i1.35410

García, L., y Nora, D. (2010). COMPETENCIAS BÁSICAS Tutores en Educación Superior: El desafío de un rol a construir. Congreso Iberoamericano De educación. (p.1-7). Buenos Aires: Universidad de Buenos Aires. Facultad de Psicología. Recuperado de https://www.adeepra.org.ar/congresos/Congreso\%20IBEROAMERICANO/COMPETENCIASBASICAS/RLE2568_Labandal.pdf

Galaz, A. (2011). Estrategias Reflexivas. La formación de profesores y de formadores de profesores https://humanidades.uach.cl/publicacion/estrategias-reflexivas-la-formacion-de-profesores-y-de-formadores-de-profesores/ LOM - Universidad Austral de Chile

Damián, E y Villarroel, L. (2010). Inteligencia emocional y desempeño docente en el proceso de enseñanza- aprendizaje (Tesis para optar el grado de magister). Lima: Universidad Enrique Guzmán y Valle.

Goleman, D. (1995). La inteligencia emocional. Barcelona: Kairos

Goleman, D. (2004). La inteligencia emocional en la empresa. Más allá de la pericia aptitudes de las estrellas. http://fcioga.org/Portals/0/Practicant/Bibilioteca/Goleman. Daniel-Inteligencia_Emocional_en_la_Empresa.pdf p.44 
Hernández R, Fernández C. y Baptista M (2014). Metodología de la Investigación (6ta ed.) México: Ediciones Mc Graw-Hill.

Lázaro, A. (1997). La acción tutorial de la función docente universitaria: Revista Complutense de Educación, vol. 8, 232-252.

Martínez, M. (2016). La formación inicial de los maestros: una responsabilidad compartida. Sociedad Española de Pedagogía. Bordón 68 (2),9-16. Doi: https://10.13042/ Bordon.2016.68201

Molero, D., Pantoja-Vallejo, A., \& Galiano-Carrión, M. (2017). Inteligencia emocional rasgo en la formación inicial del profesorado. Contextos Educativos. Revista de Educación, 0(20), 43-56. doi:https://doi.org/10.18172/con.2993

Pérez, A. (2012). inteligencia emocional y motivación del estudiante universitario (Tesis para optar el grado de doctor). Las Palmas de Gran Canaria.

Puertas Molero, P., Ubago Jiménez, J., Moreno Arrebola, R., Padial Ruz, R., Martínez Martínez, A., \& González Valero, G. (2018). La inteligencia emocional en la formación y desempeño docente: una revisión sistemática. REOP - Revista Española de Orientación y Psicopedagogía, 29(2), 128-142. doi:https://doi.org/10.5944/reop. vol.29.num.2.2018.23157

Ros Martínez, A., \& Garcés de los Fayos Ruiz, E. J. (2013). Inteligencia emocional y deporte: situación actual del estado de la investigación. Cuadernos De Psicología Del Deporte, 13(1), 105-112. Recuperado a partir de https://revistas.um.es/cpd/article/view/177241

Zarate, D. (2012). Liderazgo directivo y el desempeño docente en instituciones educativas de primaria del distrito de Independencia (Tesis para optar el grado de magister). Universidad Nacional Mayor de San Marcos. Lima. 